American Journal of Applied Sciences 6 (4): 757-771, 2009

ISSN 1546-9239

(C) 2009 Science Publications

\title{
The Convergence-Confinement Method: Roles and Limits in Modern Geomechanical Tunnel Design
}

\author{
Pierpaolo Oreste \\ Department of Land, Environmental and Geotechnologies Engineering, \\ Politecnico of Turin, Corso Duca degli Abruzzi 24, 10129 Turin, Italy
}

\begin{abstract}
The convergence-confinement method, based on the analysis of the stress and strain state that develops in the rock around a circular tunnel, has been presented in this study with the two analytical solutions that have been most frequently used in recent years. Problem statement: Thanks to the increased power of electronic processors and the spread of numerical methods, the role of the convergence-confinement method had changed but nevertheless remains important in the geo mechanical design of tunnels. Approach: Apart from being a basic instrument for the preliminary design of support and reinforcement structures, it can be profitably used for the estimation of the loads that act on the support structures, of the thickness of the plastic zone at the tunnel boundary, of the expected convergences and it can also be used to obtain an estimation of the geo mechanical parameters of the rock mass, through back-analysis staring from the in situ measurements. Conclusion: This study illustrated the fields in which the method still presents important practical applications and also its greatest limits, in more detail.
\end{abstract}

Key words: Tunnel wall displacement, rock-support interaction, elasto-plastic behaviour, deep circular tunnel

\section{INTRODUCTION}

There are at present three different types of approach that are today used in tunnel design: The empirical, rational and observational approaches.

The empirical approach is characterised by a set of design recommendations and intervention schemes that derive from experience and it very often refers to the geo mechanical characterisation of the rocks and soils involved in tunnel construction. Geo mechanical classifications are widely used, for example, in the excavation of tunnels. Today this type of approach is only used in the preliminary design stages when it is necessary to have general indications that are able to orient the following detailed study in a more efficient manner.

The rational approach uses analytical solutions, when they are available and numerical calculation methods to evaluate the stress and strain state in the rock and in the support and reinforcement structures. The purpose of this approach is to define the static conditions of the tunnel that is being examined and to design the structures that are necessary to guarantee stability to the void.

The observational approach foresees the monitoring of a work during its construction as an integral part of the project: The necessary interventions are defined or validated on the basis of the interpretation of the measurement results and therefore on the basis of the response that the surrounding medium supplies in answer to the construction of the tunnel. Recently the use of sophisticated mathematical techniques, called backanalysis techniques, has permitted a more rational organisation of the information that is obtained from the monitoring of a work and therefore the exploitation of the measurements carried out during the work construction to a greater extent.

The three previously mentioned approaches are not today considered as alternatives but rather as being complementary to each other. Each approach allows one to add useful information to the geo mechanical design of a tunnel.

The convergence-confinement method ${ }^{[1-4]}$ is part of the rational approach and uses an analytical type calculation. It is based on the analysis of the stress and strain state that develops in the rock around a tunnel. The simplicity of the method, a characteristic that has led to its widespread use since the end of the seventies, is due to the important hypotheses on which it is based:

- Circular and deep tunnels (boundary conditions of the problem to infinity)

- Lithostatic stresses of a hydrostatic type and constant in the surrounding medium of the tunnel (the variation of the stresses with depth due to the weight of the rock is neglected) 
- Continuous, homogeneous and isotropic rock mass

- Bi-dimensional problem and plane stress field

Thanks to the increased power of electronic processors and the spread of numerical methods, the role of the convergence-confinement method has changed but nevertheless remains important in the geo mechanical design of tunnels. Apart from being a basic instrument for the preliminary design of support and reinforcement structures, it can be profitably used for the estimation of the loads that act on the support structures, of the thickness of the plastic zone at the tunnel boundary, of the expected convergences and it can also be used to obtain an estimation of the geo mechanical parameters of the rock mass, through backanalysis staring from the in situ measurements.

After having presented the two analytical solutions of the convergence-confinement method that have taken on greatest importance over the years, this research illustrates the fields in which the method still presents important practical applications and also its greatest limits, in more detail.

\section{MATERIAL AND METHODS}

The convergence-confinement curve (ground reaction curve): The convergence-confinement method basically consists of the definition of the internal pressure (radial stress)-radial displacement (in absolute values) (p-lul) relationships on the boundary of a circular void that represents the tunnel. This relationship is called the convergence-confinement curve (Fig. 1). For an internal pressure equal to $\mathrm{p}_{0}$ (lithostatic stress) there is no change in the initial stress and strain state around the void and therefore the radial displacement of the wall is nil. With a diminishing of the internal pressure $\mathrm{p}$, the radial displacement of the wall begins to appear: Initially this increases linearly. At a certain point (for pressures lower than $\mathrm{p}_{\mathrm{cr}}$ ), the trend can result to be of a curvilinear type. In the simplest case of rock behaviour of an elastic type, the convergence-confinement curve is represented by a linear segment.

There are five equations that govern the elastic medium around the void:

- The two stress-strain laws according to the elasticity theory (plane stress field: $\varepsilon_{\perp}=0$ ) (Eq. 1)

- The equilibrium equation of the forces under axialsymmetrical conditions (Eq. 2 and Fig. 2)

- The two strain congruence equations under axialsymmetric conditions (Eq. 3)

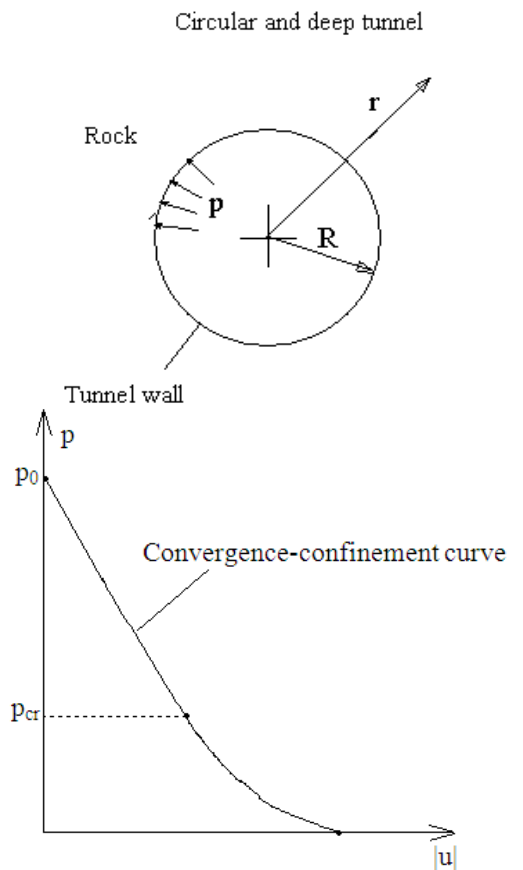

Fig. 1: Convergence-confinement method: Geometry of the problem and example of a convergenceconfinement curve. Key: p: Internal tunnel pressure, R: Tunnel radius, r: Radial coordinate, u: Radial displacement of the tunnel, $\mathrm{p}_{\mathrm{o}}$ : Lithostatic stress, $\mathrm{p}_{\mathrm{er}}$ : Critical pressure

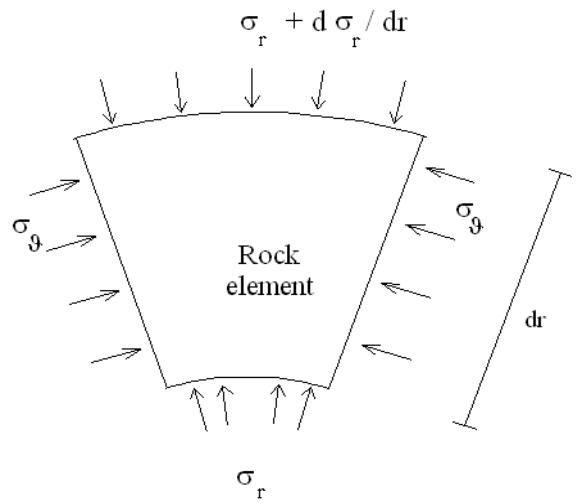

Fig. 2: Equilibrium of the forces of an infinitesimal element around the void in axial-symmetric conditions

$\left\{\begin{array}{l}\varepsilon_{\mathrm{r}}=\mathrm{a} \cdot \sigma_{\mathrm{r}}-\mathrm{b} \cdot \sigma_{\vartheta} \\ \varepsilon_{\vartheta}=\mathrm{a} \cdot \sigma_{\vartheta}-\mathrm{b} \cdot \sigma_{\mathrm{r}}\end{array}\right.$

With: $a=\frac{1}{E}-\frac{v^{2}}{E}, b=\frac{v+v^{2}}{E}$ 
Where:

$$
\begin{aligned}
\sigma_{\mathrm{r}} \text { and } \sigma_{\vartheta}= & \text { The radial and circumferential stresses } \\
& \text { (the compression stresses being } \\
& \text { considered positive) these also } \\
& \text { represent the main stresses }
\end{aligned}
$$

$\varepsilon_{\mathrm{r}}, \varepsilon_{\vartheta}$ and $\varepsilon_{\perp}=$ The radial, circumferential and longitudinal strains (along the tunnel axis)

$\mathrm{E}$ and $v \quad=$ The elastic modulus and the Poisson ratio of the rock

$\frac{\mathrm{d} \sigma_{\mathrm{r}}}{\mathrm{dr}}=\frac{\sigma_{\vartheta}-\sigma_{\mathrm{r}}}{\mathrm{r}}$

$$
\left\{\begin{array}{l}
\varepsilon_{\vartheta}=-\frac{u_{r}}{r} \\
\varepsilon_{r}=-\frac{d u_{r}}{d r}
\end{array}\right.
$$

Where, $u_{r}$ is the radial displacement of a point at a distance $r$ from the tunnel axis.

The boundary conditions of the problem are as follows:

- For $r=\infty \sigma_{\mathrm{r}}=\mathrm{p}_{0}, \sigma_{\vartheta}=\mathrm{p}_{0}, \varepsilon_{\mathrm{r}}=0, \varepsilon_{\vartheta}=0$

- For $r=R: \sigma_{r}=p$

From Eq. 1-3 and by setting the previously mentioned boundary conditions, one obtains the equations that describe the trend of the strains Eq. 4 and 5, the stresses Eq. 6 and 7 and the radial displacements Eq. 8 in the elastic field in function of the distance $r$ and the internal pressure $\mathrm{p}$ :

$$
\begin{aligned}
& \varepsilon_{r}=-\frac{1+v}{E}\left(p_{0}-p\right) \cdot\left(\frac{R}{r}\right)^{2} \\
& \varepsilon_{\vartheta}=\frac{1+v}{E}\left(p_{0}-p\right) \cdot\left(\frac{R}{r}\right)^{2} \\
& \sigma_{r}=p_{0}-\left(p_{0}-p\right) \cdot\left(\frac{R}{r}\right)^{2} \\
& \sigma_{\vartheta}=p_{0}+\left(p_{0}-p\right) \cdot\left(\frac{R}{r}\right)^{2}
\end{aligned}
$$

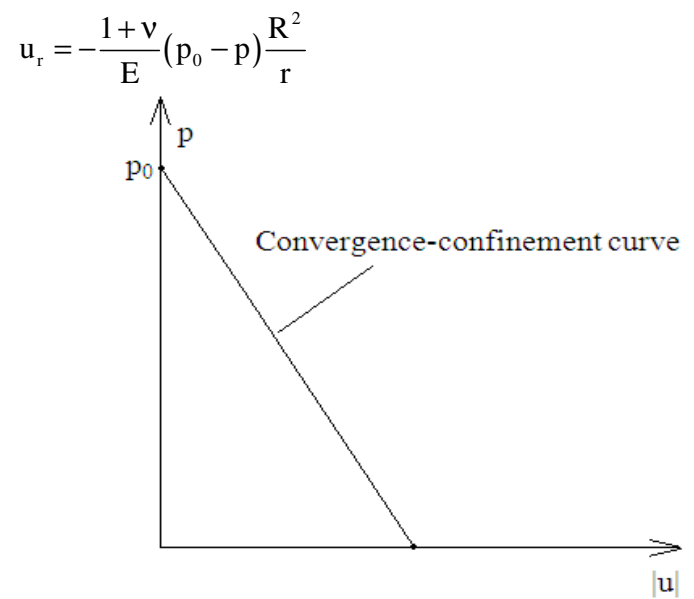

Fig. 3: Convergence-confinement curve of a tunnel for a linear-elastic medium

By placing $\mathrm{r}=\mathrm{R}$ in Eq. 8 one obtains the convergence-confinement curve (Fig. 3):

$\mathrm{u}=-\frac{1+\mathrm{v}}{\mathrm{E}}\left(\mathrm{p}_{0}-\mathrm{p}\right) \cdot \mathrm{R}$

In the more general case of rock with elastic-plastic behaviour, the elastic limit of the rock is reached when, for a certain internal pressure $p$ and with the decreasing of $r$, the stress state reaches the limit conditions defined by the strength criterion. Such a value of $r$ is called the plastic radius $R_{\mathrm{pl}}$. The radial pressure on the plastic radius is called the critical pressure $p_{c r}$, which is only a function of the peak strength parameters of the rock mass and is therefore independent of $\mathrm{R}_{\mathrm{pl}}$. For the MohrCoulomb strength criterion, for example ${ }^{[5]}$.

$\mathrm{p}_{\mathrm{cr}}=\mathrm{p}_{0} \cdot\left(1-\operatorname{sen} \varphi_{\mathrm{p}}\right)-\mathrm{c}_{\mathrm{p}} \cdot \cos \varphi_{\mathrm{p}}$

where, $c_{p}$ and $\varphi_{p}$ are the peak cohesion and friction angle.

While, for the Hoek and Brown strength criterion $^{[6]}$ :

$\mathrm{p}_{\mathrm{cr}}=\mathrm{p}_{0}-\sigma_{\mathrm{c}} \cdot \mathrm{M}$

Where:

$$
\mathrm{M}=\frac{1}{2} \cdot \sqrt{\left(\frac{\mathrm{m}_{\mathrm{p}}}{4}\right)^{2}+\mathrm{m}_{\mathrm{p}} \cdot \frac{\mathrm{p}_{0}}{\sigma_{\mathrm{c}}}+\mathrm{s}_{\mathrm{p}}}-\frac{\mathrm{m}_{\mathrm{p}}}{8}
$$

$\mathrm{m}_{\mathrm{p}}$ and $\mathrm{s}_{\mathrm{p}}=$ The peak strength parameters 
$\sigma_{\mathrm{c}}$

$=$ The uniaxial compression strength of the
intact rock

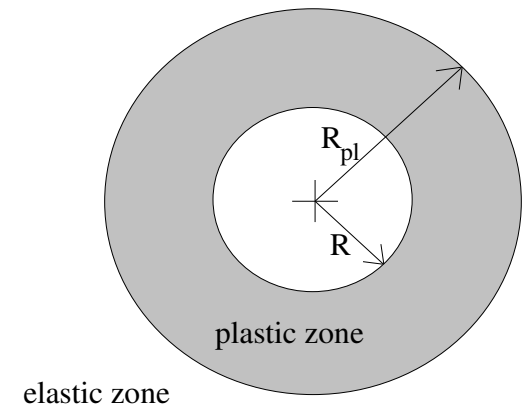

Fig. 4: The plastic zone around the tunnel

If $\mathrm{p}_{\mathrm{cr}}$ results to be negative or equal to zero, the convergence-confinement curve continues to appear as a linear segment, that is, though the material has an elastic-plastic behaviour, the elastic limit is not reached in any point and the material remains in the elastic field throughout. Instead if $\mathrm{p}_{\mathrm{cr}}$ is positive, a zone of thickness $\left(\mathrm{R}_{\mathrm{pl}}-\mathrm{R}\right)$ under plastic behaviour appears for $\mathrm{p}<\mathrm{p}_{\mathrm{cr}}$ around the void (Fig. 4). The plastic radius therefore identifies the limit of the area under plastic behaviour: The material continues to remain in elastic conditions for any distance greater than $\mathrm{R}_{\mathrm{pl}}$.

In the case where an elastic-plastic ideally brittle behaviour law can be assumed, the stress state in the plastic zone respects the residual strength criterion. By substituting the expression of the strength criterion in the residual conditions (formulated in terms of main stresses $)$ in Eq. 2 instead of $\left(\sigma_{\vartheta}-\sigma_{\mathrm{r}}\right)$ and integrating with the usual boundary conditions to the void perimeter (for $\left.r=R: \sigma_{r}=p\right)$ the following expressions of the radial stress in the plastic zone are obtained.

For the Mohr-Coulomb strength criterion:

$\sigma_{\mathrm{r}}=\left(\mathrm{p}+\frac{\mathrm{c}_{\mathrm{r}}}{\operatorname{tg} \varphi_{\mathrm{r}}}\right) \cdot\left(\frac{\mathrm{r}}{\mathrm{R}}\right)^{\frac{2 \operatorname{sen} \varphi_{\mathrm{r}}}{1-\operatorname{sen} \varphi_{\mathrm{r}}}}-\frac{\mathrm{c}_{\mathrm{r}}}{\operatorname{tg} \varphi_{\mathrm{r}}}$

where, $c_{\mathrm{r}}$ and $\varphi_{\mathrm{r}}$ : Residual cohesion and friction angle.

For the Hoek and Brown strength criterion:

$\sigma_{r}=\frac{m_{r} \cdot \sigma_{c}}{4} \cdot \ln ^{2}\left(\frac{r}{R}\right)+\sqrt{m_{r} \cdot \sigma_{c} \cdot p+s_{r} \cdot \sigma_{c}^{2}} \cdot \ln \left(\frac{r}{R}\right)+p$

where, $\mathrm{m}_{\mathrm{r}}$ and $\mathrm{s}_{\mathrm{r}}$ : Residual strength parameters.

The circumferential stress in the plastic zone is therefore simply obtained through the residual strength criterion, once the radial stress is known.
The expression of the plastic radius is obtained by equalising Eq. 12 or 13 with equation 6 and solving the unknown $r$.

For the Mohr-Coulomb strength criterion:

$$
\mathrm{R}_{\mathrm{pl}}=\mathrm{R} \cdot\left[\frac{\left(\mathrm{p}_{0}+\frac{\mathrm{c}_{\mathrm{r}}}{\operatorname{tg} \varphi_{\mathrm{r}}}\right)-\left(\mathrm{p}_{0}+\frac{\mathrm{c}_{\mathrm{p}}}{\operatorname{tg} \varphi_{\mathrm{p}}}\right) \cdot \operatorname{sen} \varphi_{\mathrm{p}}}{\mathrm{p}+\frac{\mathrm{c}_{\mathrm{r}}}{\operatorname{tg} \varphi_{\mathrm{r}}}}\right]^{\frac{1}{\mathrm{~N}_{\Phi}-1}}
$$

Where:

$\mathrm{N}_{\Phi}^{\mathrm{r}}=\frac{1+\operatorname{sen} \varphi_{\mathrm{r}}}{1-\operatorname{sen} \varphi_{\mathrm{r}}}$

For the Hoek and Brown strength criterion:

$\mathrm{R}_{\mathrm{pl}}=\mathrm{R} \cdot \mathrm{e}^{\frac{2 \cdot \mathrm{N}}{\mathrm{m}_{\mathrm{r}} \cdot \sigma_{\mathrm{c}}}}$

Where:

$$
\begin{aligned}
\mathrm{N}= & \sqrt{\mathrm{m}_{\mathrm{r}} \cdot \sigma_{\mathrm{c}} \cdot \mathrm{p}_{0}+\mathrm{s}_{\mathrm{r}} \cdot \sigma_{\mathrm{c}}^{2}-\mathrm{m}_{\mathrm{r}} \cdot \sigma_{\mathrm{c}}^{2} \cdot \mathrm{M}} \\
& -\sqrt{\mathrm{m}_{\mathrm{r}} \cdot \sigma_{\mathrm{c}} \cdot \mathrm{p}+\mathrm{s}_{\mathrm{r}} \cdot \sigma_{\mathrm{c}}^{2}}
\end{aligned}
$$

It can be noticed how, for $\mathrm{p}=\mathrm{p}_{\mathrm{cr}}$, the plastic radius coincides with the radius of the void and the plastic zone has therefore nil thickness. For lower internal pressures, $\mathrm{R}_{\mathrm{pl}}$ begins to increase and the plastic zone increases in thickness.

For values of $r$ greater than the plastic radius, the rock maintains an elastic behaviour, as described by Eq. 16-20, that are similar to Eq. 4-8, having however placed a new boundary condition in correspondence to the plastic radius:

$$
\text { For } r=\mathrm{R}_{\mathrm{pl}}: \sigma_{\mathrm{r}} \mathrm{p}_{\mathrm{cr}} \text { : }
$$

$\varepsilon_{\mathrm{r}}=-\frac{1+\mathrm{v}}{\mathrm{E}}\left(\mathrm{p}_{0}-\mathrm{p}_{\mathrm{cr}}\right) \cdot\left(\frac{\mathrm{R}_{\mathrm{pl}}}{\mathrm{r}}\right)^{2}$

$\varepsilon_{\vartheta}=\frac{1+v}{E}\left(p_{0}-p_{c r}\right) \cdot\left(\frac{R_{p l}}{r}\right)^{2}$

$\sigma_{\mathrm{r}}=\mathrm{p}_{0}-\left(\mathrm{p}_{0}-\mathrm{p}_{\mathrm{cr}}\right) \cdot\left(\frac{\mathrm{R}_{\mathrm{pl}}}{\mathrm{r}}\right)^{2}$ 
$\sigma_{\vartheta}=\mathrm{p}_{0}+\left(\mathrm{p}_{0}-\mathrm{p}_{\mathrm{cr}}\right) \cdot\left(\frac{\mathrm{R}_{\mathrm{pl}}}{\mathrm{r}}\right)^{2}$

$\mathrm{u}_{\mathrm{r}}=-\frac{1+\mathrm{v}}{\mathrm{E}}\left(\mathrm{p}_{0}-\mathrm{p}_{\mathrm{cr}}\right) \cdot \frac{\mathrm{R}_{\mathrm{pl}}^{2}}{\mathrm{r}}$

The detailed analysis of the strain behaviour in the plastic zone also allows one to obtain the expression of the radial displacement of the tunnel wall for $\mathrm{p}<\mathrm{p}_{\mathrm{cr}}$.

With the hypothesis of the Mohr-Coulomb strength criterion and considering the expression of the plastic potential of the same type as the strength criterion, it is possible to obtain a rigorous solution in closed form of the radial displacement $\mathrm{u}$ of the tunnel wall for $\mathrm{p}<\mathrm{p}_{\mathrm{cr}}$, that is, for $R_{p l}>R^{[5]}$ (Eq. 21):

$$
\begin{aligned}
& \mathrm{u}=-\frac{1+v}{\mathrm{E}} \cdot\left\{\frac{\mathrm{R}_{\mathrm{pl}}^{\mathrm{N}_{\Psi}+1}}{\mathrm{R}^{N_{\Psi}}} \cdot\left(\mathrm{p}_{0}+\frac{\mathrm{c}_{\mathrm{p}}}{\operatorname{tg} \varphi_{\mathrm{p}}}\right) \cdot \operatorname{sen} \varphi_{\mathrm{p}}+\right. \\
& (1-2 \cdot v) \cdot\left(\mathrm{p}_{0}+\frac{\mathrm{c}_{\mathrm{r}}}{\operatorname{tg} \varphi_{\mathrm{r}}}\right) \cdot\left(\frac{\mathrm{R}_{\mathrm{pl}}^{N_{\mathrm{p}}+1}}{\mathrm{R}^{N_{\Psi}}}-\mathrm{R}\right) \\
& \frac{-\left[1+\mathrm{N}_{\Phi}^{\mathrm{r}} \cdot \mathrm{N}_{\Psi}-v \cdot\left(\mathrm{N}_{\Psi}+1\right) \cdot\left(\mathrm{N}_{\Phi}^{\mathrm{r}}+1\right)\right]}{\left(\mathrm{N}_{\Phi}^{\mathrm{r}}+\mathrm{N}_{\Psi}\right) \cdot \mathrm{R}^{\left(\mathrm{N}_{\Phi}^{\mathrm{r}}-1\right)}} \\
& \left.\cdot\left(\mathrm{p}+\frac{\mathrm{c}_{\mathrm{r}}}{\operatorname{tg} \varphi_{\mathrm{r}}}\right) \cdot\left(\frac{\mathrm{R}_{\mathrm{pl}}^{\left(\mathrm{N}_{\Phi}^{\mathrm{r}}+\mathrm{N}_{\Psi}\right)}}{\mathrm{R}^{N_{\Psi}}}-\mathrm{R}^{\mathrm{N}_{\Phi}^{\leftarrow}}\right)\right\}
\end{aligned}
$$

Where:

$$
\begin{aligned}
& \mathrm{N}_{\Psi}=\frac{1+\operatorname{sen} \psi}{1-\operatorname{sen} \psi} \\
& \psi=\text { Dilatancy }
\end{aligned}
$$

It is also possible to describe the convergenceconfinement curve of the tunnel in closed form for the Hoek and Brown strength criterion for $\mathrm{p}<\mathrm{p}_{\mathrm{cr}}$, but only by making some simplifications to the mathematical treatment of the strains in the plastic field (Fig. 5) ${ }^{[6]}$ :

$$
u=-\frac{2 \cdot R \cdot(1+v)}{E} \cdot M \cdot \frac{\sigma_{c}}{(f+1)} \cdot\left[\frac{f-1}{2}+\left(\frac{R_{p l}}{R}\right)^{f+1}\right]
$$

where, f: The parameter that describes the strain behaviour of the rock mass in the plastic field ( $\mathrm{f}>1$ ).

The most important adopted simplification consists in considering the total deformations in the plastic field (and not the elastic component which is governed by elastic parameters) separately from the plastic component (which is governed by the plastic potential).
In both cases (M-C or H-B strength criterion), the parameters that characterise the strain behaviour in the plastic field ( $\psi$ or $f)$ influence the convergenceconfinement curve of the tunnel to a great extent. Their determination is however not easy: No empirical rules exist to facilitate this evaluation nor are there any suitable in situ tests for this purpose.
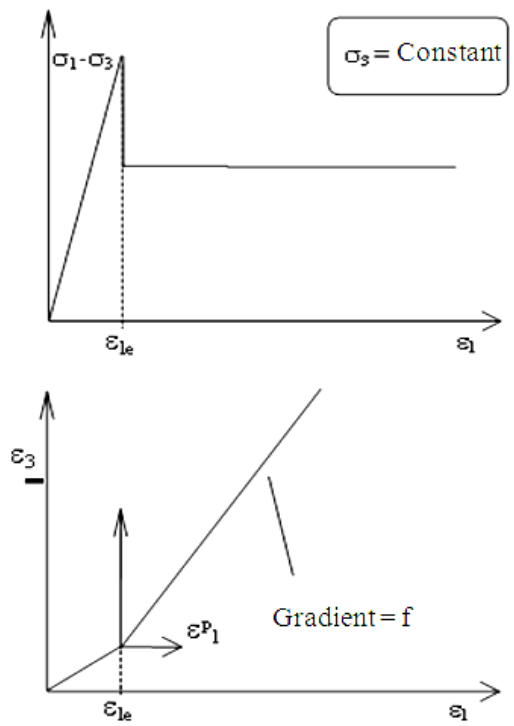

Fig. 5: Relationship of the total main strains in the plastic field, generally assumed when Hoek and Brown's strength criterion is adopted

An interesting way of obtaining the estimation of these parameters is that of proceeding with back-analysis of the convergence measurements that become available during the construction of the research.

The previously described analytical formulation of the convergence-confinement curve is usually integrated with a correction to take the overload produced on the tunnel roof by the weight of the plastic rock itself into consideration. Such a correction is obviously accomplished for pressures that are lower than $\mathrm{p}_{\mathrm{cr}}$ and consists in the increasing of the value of $\mathrm{p}$ of each point of the curve by a value of $\Delta \mathrm{P}=\gamma$. $\left(\mathrm{R}_{\mathrm{pl}}-\mathrm{R}\right)$. In this way the trend of the convergence-confinement curve can, at a certain point, show the tendency of becoming distant from the axis of the displacements.

Simulation of the support structure: The convergence-confinement curve that was described previously does not take any possible support structure in the tunnel into consideration, but only considers a generic internal pressure which, varying, provokes a 
different tunnel response both in terms of convergence and in terms of extension of the plastic zone. In order to analyse the interaction between the tunnel and the support structures in more detail, it is necessary to represent the support structures through their own reaction line and to introduce the concept of fictitious internal pressure.

The fictitious internal pressure is a quantity that is introduced in order to allow one to face a threedimensional problem (due to the presence of the excavation face) with a simplified bi-dimensional scheme as in fact the convergence-confinement method is. After having identified a precise section to study along the tunnel axis, the following different situations are taken into consideration:

- When the excavation face is still very far from the studied section, the internal pressure acting on the perimeter of the future tunnel is equal to $\mathrm{p}_{0}$ : The stress perturbation produced by the excavation works at the studied section can be considered negligible (Fig. 6a)

- As the excavation face comes closer to the studied section, a certain stress release is produced ahead of the face which involves a reduction of the internal pressure and the appearance of radial displacements on the perimeter of the future tunnel even before it is excavated (Fig. 6b)

- When the face passes the studied section, the contribution offered by the face to the static of the tunnel can be taken into consideration through the fictitious internal pressure concept which diminishes, until it disappears, in function of the distance from the tunnel face (Fig. 6d-e)

With the concept of the fictitious internal pressure, it is therefore possible to consider the convergenceconfinement curve as a graphic representation of the strain situation that is produced along the tunnel axis. Each point of the curve represents the situation in a particular section with reference to the position of the excavation face.

Panet and Guenot ${ }^{[7]}$ and later A.F.T.E.S. ${ }^{[8]}$ (Eq. 23) suggested the analytical formulation of the trend of the radial displacements on the tunnel perimeter both ahead and behind the excavation face for the particular case of a tunnel in an elastic medium and without supports. From this curve and knowing the convergenceconfinement curve of the tunnel in elastic conditions, it is possible to obtain the expression of the fictitious internal pressure along the tunnel axis, for the zone behind the excavation face this is: $u_{x}=u_{\infty} \cdot\left[1-a \cdot\left(\frac{a \cdot R}{x+a \cdot R}\right)^{2}\right]$

$\mathrm{p}_{\text {fict }}=\mathrm{a} \cdot \mathrm{p}_{0} \cdot \frac{\mathrm{b}}{\mathrm{x}+\mathrm{b}}$

Where:

$\mathrm{a}=0,75$

$\mathrm{x}=$ Distance from the face

$\mathrm{u}_{\infty}=$ Radial displacement of the wall at a great distace from the face $(x=\infty)$

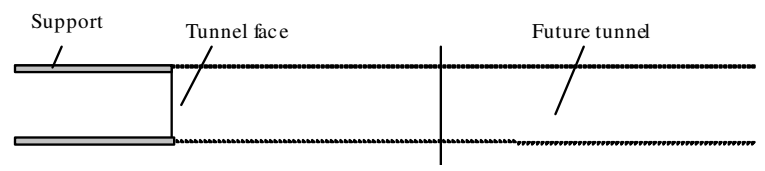

Studied section

(a)

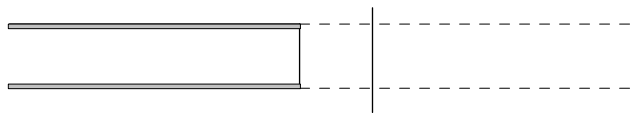

(b)

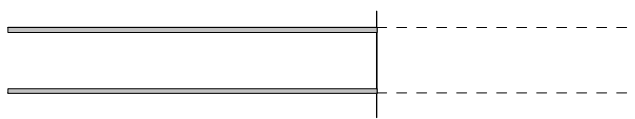

(c)

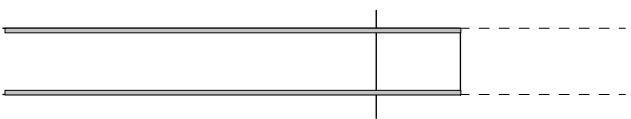

(d)

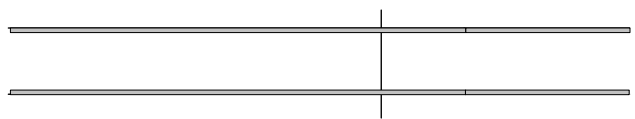

(e)

Fig. 6: Location of the excavation face with respect to the considered studied section

This expression indicates that, in the hypothesised particular situation, a fictitious internal pressure that is equal to $(75 \%)$ of the lithostatic stress $\mathrm{p}_{0}$ exists in correspondence to the excavation face $(x=0)$.

For an elastic-plastic medium and in the presence of support structures, the trend of the radial displacements along the tunnel axis doesn't vary substantially, even though varies the $u_{\infty}$ value. The fictitious internal pressure, in correspondence to the excavation face, is however usually lower than the value identified for the hypothesis of an elastic medium without supports (Eq. 23).

One of the most interesting aspects of the convergence-confinement method is that it is possible 
to also take the presence of supports into consideration through the distinct definition of their reaction line (Fig. 7) ${ }^{[3,6,8]}$.

The supports are installed close to the excavation face where the tunnel wall has already shown a certain radial displacement $\left(\mathrm{u}_{\mathrm{in}}\right)$. The value $\mathrm{u}_{\mathrm{in}}$ can be obtained iteratively on the basis of Eq. 23 that can be rewritten in the following terms:

$$
\frac{\mathrm{u}_{\text {in }}}{\mathrm{u}_{\infty}}=\frac{\mathrm{u}_{\text {in }}}{\mathrm{u}_{\mathrm{eq}}} \cong\left[1-\mathrm{a} \cdot\left(\frac{\mathrm{a} \cdot \mathrm{R}}{\mathrm{d}+\mathrm{a} \cdot \mathrm{R}}\right)\right]
$$

Where:

$\mathrm{d}=$ The distance from the face of the section in which the support are installed

$\mathrm{u}_{\mathrm{in}}=$ Increased from the nil value until the previous equation is verified.

In the presence of the support structures, any further development of the radial displacement of the wall would provoke the loading of the supports, according to a usually linear relation that is characterised by the angular coefficient k, known as the support stiffness. The expressions of stiffness $\mathrm{k}$ of traditional supports (steel ribs, cast concrete linings and radial boltings with end anchorages) can be found in literature $^{[6]}$.

When several types of supports react at the same time, the stiffness of the system is simply given by the sum of the stiffnesses of the individual supports:

$\mathrm{k}_{\mathrm{tot}}=\sum_{\mathrm{i}} \mathrm{k}_{\mathrm{i}}$

Where:

$\mathrm{k}_{\mathrm{tot}}=$ The total stiffness of the support structure

$\mathrm{k}_{\mathrm{i}}=$ The stiffness of an individual support

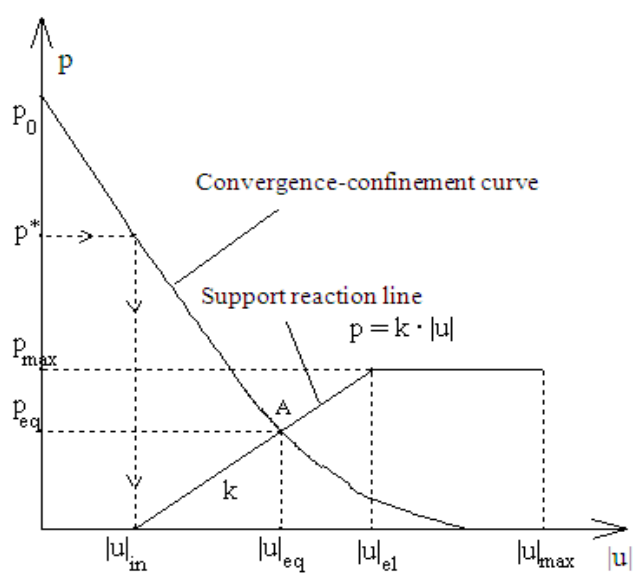

Fig. 7: Convergence-confinement curve of a tunnel and reaction ine of the supports. Key: $\mathrm{p}^{*}$ and $\mathrm{u}_{\mathrm{in}}$ : Fictitious internal pressure and radial displacement of the wall where the supports are installed, $\mathrm{p}_{\max }$ : Maximum pressure that the supports can bear, $\mathrm{p}_{\mathrm{eq}}$ and $\mathrm{u}_{\mathrm{eq}}$ : Load on the supports and radial displacement of the wall in the final equilibrium condition $\left(\mathrm{u}_{\mathrm{eq}}=\mathrm{u}_{\infty}\right)$, $\mathrm{u}_{\mathrm{el}}$ : Radial displacement of the wall when the supports reach the elastic limit, $\mathrm{u}_{\max }$ : Radial displacement of the wall that provokes breakage of the supports, k: Support stiffness (force length ${ }^{-3}$ )

The intersection between the convergenceconfinement curve of the tunnel and the reaction line of the supports allows one to obtain the pressure $p_{\text {eq }}$ that acts on the supports and the radial displacement $\mathrm{u}_{\mathrm{eq}}$ of the tunnel in the final equilibrium situation (at a great distance from the excavation face, $\mathrm{u}_{\mathrm{eq}}=\mathrm{u}_{\infty}$ ).

In the case of different supports at the same time, the load $p_{i}$ that acts on the generic support is a function of the displacement $\left(\mathrm{u}_{\mathrm{eq}}-\mathrm{u}_{\mathrm{in}}\right)$ and of its own stiffness $\mathrm{k}_{\mathrm{i}}$ :

$\mathrm{p}_{\mathrm{i}}=\mathrm{k}_{\mathrm{i}} \cdot\left(\mathrm{u}_{\mathrm{eq}}-\mathrm{u}_{\mathrm{in}}\right)$

It is therefore possible to compare the value of the load acting on each single support with the maximum pressure that it is able to bear.

Simulation of the rock reinforcement structures: The rock reinforcement elements that are often used in tunnels cannot be independently considered through their reaction line, as performed for the supports. These in fact are interventions that directly interact with the stress and strain behaviour of the rock around the tunnel. Radial reinforcements (bolts with continuous anchorage), for example, interact with the rock through the shear stresses that develop all along the bolt-rock interface. Thanks to their presence, axi-symmetric equilibrium Eq. 2 changes into the following expression:

$\frac{\mathrm{d} \sigma_{\mathrm{r}}}{\mathrm{dr}}=\frac{\sigma_{\vartheta}-\sigma_{\mathrm{r}}}{\mathrm{r}}+\frac{\mathrm{dT}}{\mathrm{dr}} \cdot \frac{\mathrm{R}}{\mathrm{S}_{\mathrm{L}} \cdot \mathrm{S}_{\mathrm{T}_{0}}} \cdot \frac{1}{\mathrm{R}}$

where, $\mathrm{T}$ is the tensile force in the bolts at the generic distance $r$ from the tunnel axis, which leads to a significant alteration in the distribution of the stresses and strains around the tunnel.

The tunnel convergence-confinement curve is therefore modified, due to the presence of the radial reinforcements, in the way that is shown in Fig. $8^{[9,10]}$.

The reinforcement interventions of the excavation face show a different mechanism as they do not involve the rock around the tunnel and therefore do not modify the convergence-confinement curve. On the other hand, they cannot even be taken into consideration in the 
convergence-confinement method through their reaction line. Their presence however modifies the development of the radial displacements ahead of the tunnel face: with the improvement of the mechanical characteristics of the nucleus ahead of the face, a high contrast is guaranteed on the walls of the future tunnel. The final result is that the convergences, in correspondence to the excavation face, are low (low values of $\mathrm{u}_{\mathrm{in}}$ ) and the loads applied to the supports are higher (Fig. 9).

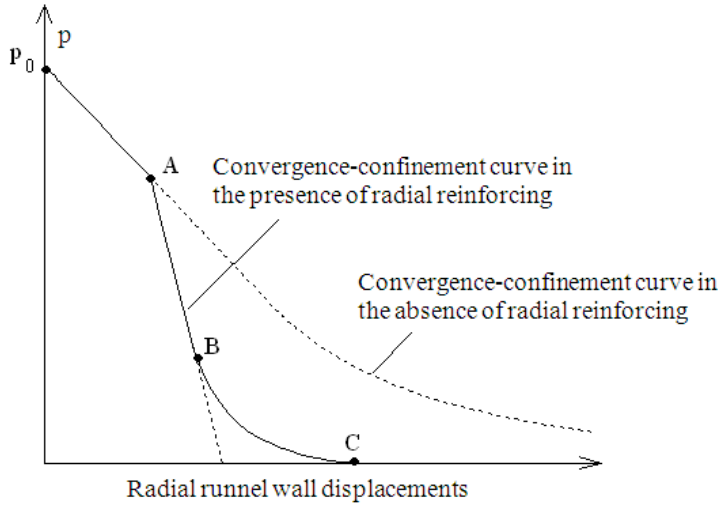

Fig. 8: A typical convergence-confinement curve of a tunnel in the presence of radial reinforcements. Key: A: Reinforcement intervention, B: Yielding of the natural rock or of the reinforced rock, C: Radial displacements of the tunnel wall without supports

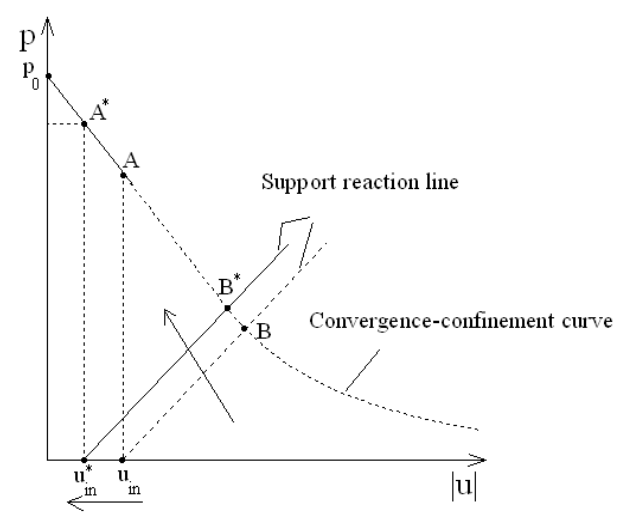

Fig. 9: Longitudinal reinforcement of the face using the convergence-confinement method. Key: A and $\mathrm{A}^{*}$ : Point of the convergence-confinement curve that refers to the situation of the transversal section in which the supports are installed, without and with the face reinforcement, B and $\mathrm{B}^{*}$ : Final equilibrium point, without and with the face reinforcement, $\mathrm{u}_{\mathrm{in}}$ and $\mathrm{u}_{\mathrm{in}}{ }^{*}$ : Radial displacement of the wall in the section in which the supports are installed, without and with the face reinforcement
Evaluation of the stress-strain condition of the face using the convergence-confinement method of a spherical void: If the convergence-confinement method is applied to a spherical void ${ }^{[11]}$ (Fig. 10) instead of to a circular geometry, the stress and strain conditions of the excavation face of a tunnel can be evaluated with a certain degree of approximation.

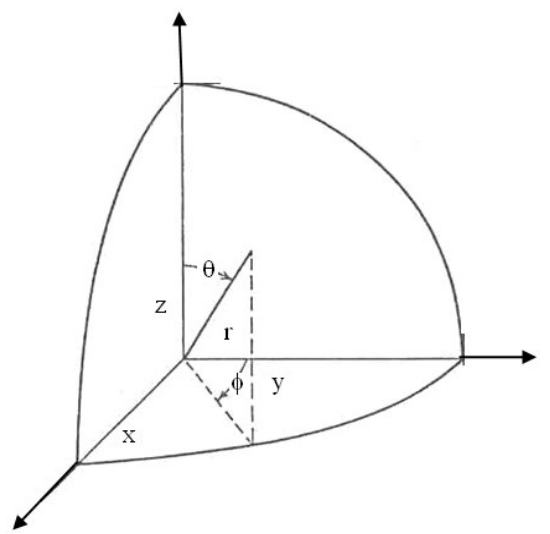

Fig. 10: A spherical void considered to evaluate excavation face conditions using the convergence-confinement method ${ }^{[12]}$

According to this statement ${ }^{[12]}$, the excavation face area is considered as a hemisphere with a diameter equal to that of the tunnel. Initially, a pressure equal to the lithostatic pressure $\mathrm{p}_{0}$ exists inside the hemisphere (before excavation works commence); later the internal pressure gradually reduces, as the excavation proceeds, until it is cancelled.

The most important results of this calculation are:

- The extrusion of the excavation face (the radial displacement of the hemisphere for a nil internal pressure)

- The extension of the plastic zone ahead of the excavation face

The analytical formulation of the convergenceconfinement method for a spherical void is similar to that of a circular geometry. Equation 27-29, which substitute Eq. 6-8, allow the stress state (radial and circumferential) and the radial displacements ahead of the face to be obtained, for the case of rock with an elastic behaviour:

$$
\sigma_{\mathrm{r}}=\mathrm{p}_{0}-\left(\mathrm{p}_{0}-\mathrm{p}\right) \cdot\left(\frac{\mathrm{R}}{\mathrm{r}}\right)^{3}
$$


$\sigma_{\vartheta}=\mathrm{p}_{0}+\frac{1}{2} \cdot\left(\mathrm{p}_{0}-\mathrm{p}\right) \cdot\left(\frac{\mathrm{R}}{\mathrm{r}}\right)^{3}$

$\mathrm{u}_{\mathrm{r}}=-\frac{1+\mathrm{v}}{2 \cdot \mathrm{E}} \cdot\left(\mathrm{p}_{0}-\mathrm{p}\right) \cdot \frac{\mathrm{R}^{3}}{\mathrm{r}^{2}}$

By placing $r=R$ and $p=0$ in Eq. 29, it is possible to obtain the extrusion of the excavation face for rock with an elastic behaviour:

$\mathrm{u}=-\frac{1+\mathrm{v}}{2 \cdot \mathrm{E}} \cdot \mathrm{p}_{0} \cdot \mathrm{R}$

The critical pressure, for the Mohr-Coulomb strength criterion, can now be calculated using the following expression, which substitutes Eq. 10:

$\mathrm{p}_{\mathrm{cr}}=\frac{\frac{3}{2} \cdot \mathrm{p}_{0} \cdot\left(1-\operatorname{sen} \phi_{\mathrm{p}}\right)-2 \cdot \mathrm{c}_{\mathrm{p}} \cdot \cos \phi_{\mathrm{p}}}{\left(\frac{3}{2}+\frac{1}{2} \cdot \operatorname{sen} \phi_{\mathrm{p}}\right)}$

The plastic radius is now evaluated using Eq. 32, which substitutes Eq. 14:

$\mathrm{R}_{\mathrm{pl}}=\mathrm{R} \cdot\left[\frac{\frac{\frac{3}{2} \cdot \mathrm{p}_{0} \cdot\left(1-\operatorname{sen} \phi_{\mathrm{p}}\right)-2 \cdot \mathrm{c}_{\mathrm{p}} \cdot \cos \phi_{\mathrm{p}}}{\left(\frac{3}{2}+\frac{1}{2} \cdot \operatorname{sen} \phi_{\mathrm{p}}\right)}+\frac{\mathrm{c}_{\mathrm{r}}}{\operatorname{tg} \phi_{\mathrm{r}}}}{\mathrm{p}+\frac{\mathrm{c}_{\mathrm{r}}}{\operatorname{tg} \phi_{\mathrm{r}}}}\right]^{\frac{1}{2 \cdot\left(N_{\mathrm{f}}^{\mathrm{r}}-1\right)}}$

For a nil pressure at the excavation face $(p=0)$ Eq. 32 is reduced to:

$\mathrm{R}_{\mathrm{pl}}=\mathrm{R} \cdot\left[\frac{\left[\begin{array}{l}\frac{3}{2} \cdot \mathrm{p}_{0} \cdot\left(1-\operatorname{sen} \phi_{\mathrm{p}}\right) \\ -2 \cdot \mathrm{c}_{\mathrm{p}} \cdot \cos \phi_{\mathrm{p}}\end{array}\right] \cdot \operatorname{tg} \phi_{\mathrm{r}}}{\left(\frac{3}{2}+\frac{1}{2} \cdot \operatorname{sen} \phi_{\mathrm{p}}\right) \cdot \mathrm{c}_{\mathrm{r}}}+1\right]^{\frac{1}{2 \cdot\left(\mathrm{N}_{\mathrm{f}}-1\right)}}$

Finally, the axial displacement of the excavation face (extrusion) for $\mathrm{p}=0$, can be calculated using Eq. 34, which substitutes Eq. 21:

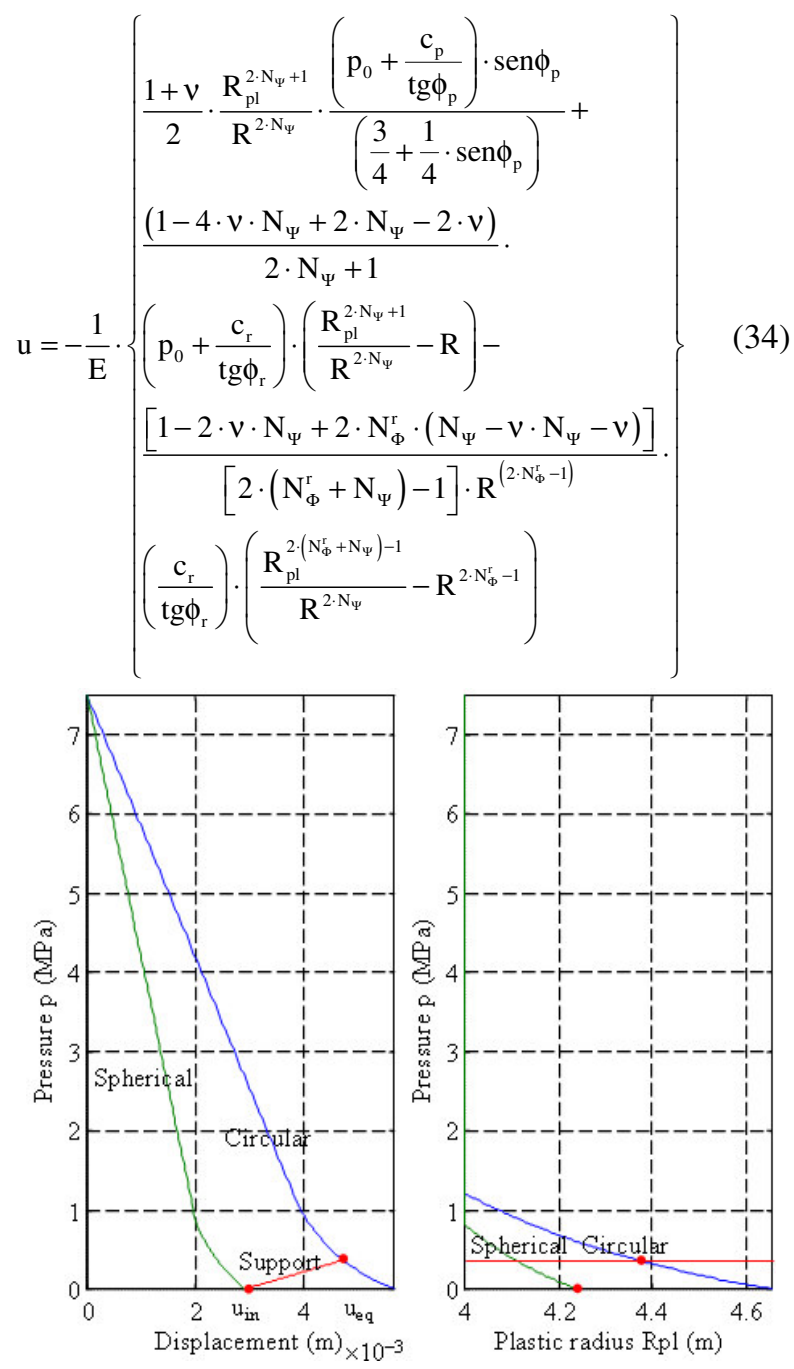

Fig. 11: Convergence-confinement curve (left) and trend of the plastic radii in function of the internal pressure (right) for a circular geometry and a spherical void, for a $4 \mathrm{~m}$ diameter and $300 \mathrm{~m}$ deep tunnel in a rock mass with a GSI quality index $=48$

In short, for the stress and strain analysis of the rock ahead of the excavation face, it is first necessary to evaluate the $p_{c r}$ in Eq. 31. If this $p_{c r}$ is equal or lower than zero, the rock ahead of the face has an elastic behaviour and it is necessary to use Eq. 30 to evaluate the extrusion of the excavation face. On the other hand, if the $p_{c r}$ is higher than zero, it is necessary to first evaluate the plastic radius using Eq. 33 and then the extrusion of the face with Eq. 34. 
Equation 30 and 34 also offer the possibility of estimating the value of the radial displacement of the tunnel walls $u_{\text {in }}$ (Fig. 7) in correspondence to the excavation face. These equations therefore allow $\mathrm{u}_{\text {in }}$ to be calculated in an alternative way to the procedure illustrated in Fig. 7. This second way of calculating $\mathrm{u}_{\mathrm{in}}$, though very practical, does not allow the effect of the supports on the radial displacements of the walls in the excavation face area to be taken into account.

Figure 11 shows, as an example, the results of the calculation with the convergence-confinement method of a circular geometry and a spherical void, for a $300 \mathrm{~m}$ deep tunnel with a $4 \mathrm{~m}$ radius in a rock mass which has a GSI geo mechanical index $=48(\mathrm{c}=0.85 \mathrm{MPa}$, $\varphi=50^{\circ}, \mathrm{E}=8250 \mathrm{MPa}, \psi=10^{\circ}$ ). The behaviour of the rock was hypothesised to be of an ideal elastic-plastic type $\left(c_{\mathrm{p}}=\mathrm{c}_{\mathrm{r}}, \varphi_{\mathrm{p}}=\varphi_{\mathrm{r}}\right)$. The support structures have a stiffness $\mathrm{k}$ of $200 \mathrm{MPa} \mathrm{m}^{-1}$.

From the graphs of Fig. 11 it is possible to obtain:

- The extrusion of the excavation face (displacement of the convergence-confinement curve of the spherical geometry for an internal pressure $p=0$ ) equal to $2.9 \mathrm{~mm}$, which also corresponds to the $\mathrm{u}_{\mathrm{in}}$ displacement

- The final equilibrium displacement $\mathrm{u}_{\mathrm{eq}}$ (radial displacement of the tunnel walls at a great distance from the excavation face) equal to $4.7 \mathrm{~mm}$

- The equilibrium pressure $\mathrm{p}_{\mathrm{eq}}$ (load acting on the support structure) equal to $0.36 \mathrm{MPa}$

- The extension of the plastic zone ahead of the excavation face (a value obtained from the pressure-plastic radius curve for the spherical geometry, for internal pressure nil) equal to the difference between the plastic radius and the radius of the sphere $(4.24-4=0.24 \mathrm{~m})$

- The extension of the plastic zone around the tunnel (a value obtained from the pressure-plastic radius curve for the circular geometry, for internal pressure $\mathrm{p}=\mathrm{p}_{\mathrm{eq}}$ ) equal to the difference between the plastic radius and the radius of the tunnel (4.37$4=0.37 \mathrm{~m}$ )

The combined analysis of the convergenceconfinement curve of the circular geometry and the spherical void is therefore able to supply a great deal of information on the static behaviour of a tunnel and of the support works.

The convconf calculation code, implemented in Matlab, is given in the appendix. This code is able to perform the combined analyses of the convergenceconfinement curve for the circular geometry and for the spherical void. The Mohr-Coulomb strength criterion is considered for the rock mass and the stress-strain law is elastic-brittle plastic (peak strength parameters different from the residual ones). The mathematical formula that is adopted is presented in Eq. 1-22, for the circular geometry and in Eq. 27-34 for the spherical void.

\section{RESULTS AND DISCUSSION}

Modern use of the convergence-confinement method: From an examination of the characteristics of the previously illustrated convergence-confinement method it can be seen how it is remarkably simple to use and versatile: it allows one to evaluate, as a first approximation, the stress and strain state in the rock and in the support structure at various distances from the excavation face and ahead the face. It is also today possible to consider the influence of the radial rock reinforcement elements and all other improving interventions around the tunnel in the calculation and also to face the study of the interaction between the reinforcement interventions and the support structures and among the support structures themselves in a rational way.

The important basic hypotheses however limit the use of the method. The impossibility of calculating the bending moments and the shear forces in the support structures, for example, make it impossible to use it directly for the designing of the supports and to evaluate surface subsidence for urban tunnels at shallow depths.

Today the convergence-confinement method is above all used preliminarily and together with more sophisticated numerical calculation methods which are more suitable to simulate the various construction stages of a tunnel or even together with the hyperstatic reaction method.

The convergence-confinement method still has an important role to play in the following main aspects of geomechanical tunnel design:

- Qualitative identification of the static behaviour of a tunnel

- Verification of the validity of the complex numerical models (initial comparison tests for the simplest problems)

- Evaluation of the relative importance of the calculation parameters for a particular problem

- Definition of the vertical load acting on the support structure

- Initial estimation of the thickness of the plastic zones around the tunnel

- Evaluation of the entity of the stress and strain perturbation around the tunnel

- Estimation of the entity of the tunnel convergences

- Optimisation of the support structures and the rock reinforcement elements 
- Back-analysis of the monitoring measurements of a tunnel with the purpose of obtaining a more reliable estimation of the mechanical parameters of the rock

- Evaluation of the static condition of the face

Qualitative identification of the static behaviour of a tunnel: An initial calculation using the convergenceconfinement method is able to clarify whether a tunnel requires systematic supports or not. Such a verification is usually performed by analysing the value of the thickness of the plastic zone and the entity of the radial strains around the tunnel, for a nil internal pressure. The thickness of the plastic zone supplies an indication of the volume of fractured rock on the roof of the excavation, while the radial strains allow one to evaluate the degree of the distension of the rock perpendicular to the tunnel wall: it is in fact the distension of the rock that causes the opening and propagation of existing discontinuities, with the consequence of making any blocks isolated from the discontinuity unstable.

The results, in terms of plastic radii, of two calculations using the convergence-confinement method for $3 \mathrm{~m}$ radius tunnels and a depth of $100 \mathrm{~m}$ $\left(\mathrm{p}_{0}=2.5 \mathrm{MPa}\right)$ excavated in rock masses with $\mathrm{RMR}$ quality indexes of 35 (case a) and 55 (case b) are shown in Fig. 12 and 13 as examples. The geo mechanical parameters of the rock masses were estimated through the relations suggested by Bieniaski ${ }^{[13]}$ and which are shown in Table 1.

From an examination of the Fig. 12 and 13 it is possible to see that, for nil internal pressures, the plastic radius reaches remarkable values $(7.5 \mathrm{~m})$ for an $\mathrm{RMR}=35$, while it is just over the excavation radius $(3.32 \mathrm{~m})$ for an RMR $=55$. The calculation with the convergence-confinement method also supplies the following results in terms of radial deformations on the tunnel walls for nil internal pressures: (34\%) for an $\mathrm{RMR}=35$ and $(2 \%)$ for an $\mathrm{RMR}=55$.

Table 1: Geomechanical parameters assumed for the rock mass considered in the calculations for RMR $=35$ (case a) and $\mathrm{RMR}=55$ (case b)

\begin{tabular}{ll}
\hline Parameter & Value \\
\hline RMR = 35: & \\
Elastic modulus $(\mathrm{MPa})$ & 4217 \\
m peak strength parameter & 1.178000 \\
s peak strength parameter & 0.000730 \\
m residual strength parameter & 0.116000 \\
s residual strength parameter & 0.000020 \\
Uniaxial compress. Strength $\sigma_{\mathrm{c}}(\mathrm{MPa})$ & 20 \\
RMR = 55: & \\
Elastic modulus $(\mathrm{MPa})$ & 13335 \\
m peak strength parameter & 2.406000 \\
s peak strength parameter & 0.006740 \\
m residual strength parameter & 0.482000 \\
s residual strength parameter & 0.000553 \\
Uniaxial compress. strength $\sigma_{\mathrm{c}}(\mathrm{MPa})$ & 40 \\
\hline
\end{tabular}

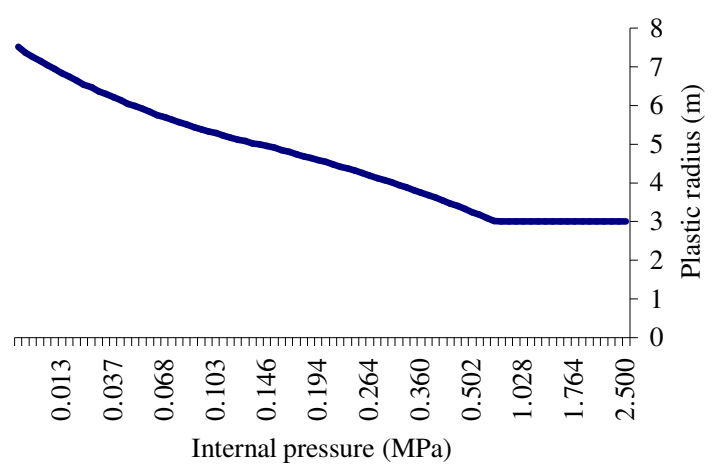

Fig. 12: Trend of the plastic radius $\mathrm{R}_{\mathrm{pl}}$ in function of the internal pressure $\mathrm{p}$ (case a)

In short, it is evident how it is possible to indicate, using the convergence-confinement method, a light and occasional support system for case b $(R M R=55)$ while a systematic and massive support system is required for case a $(\mathrm{RMR}=35)$. On the basis of these indications it is possible to decide which support to use, a choice which should however be subsequently verified using a numerical calculation.

Verification of the validity of complex numerical calculation codes: The validation of numerical calculation methods can be obtained through a comparison with the convergence-confinement method for the simple problem of a circular and deep tunnel in a hydrostatic type stress field. Because of the relative simplicity of its analytical solution, the results of the convergence-confinement method are considered correct and a numerical method that offers the same results in the case of comparison is also considered valid.

Even in the analysis of complex problems (non circular shape of the tunnel, excavation methods that require multiple-face attach and rock reinforcement interventions, stress-strain law of the rock with gradual reduction of the strength parameters from peak to residual values), it is good practice to be able to carry out a preliminary comparison between the numerical method that one intends using and the convergence-confinement method, introducing opportune simplifying hypotheses.

Evaluation of the relative importance of the calculation parameters: The geotechnical characterisation of a rock mass is always approximate, as it is based on estimations derived from empirical correlations and qualitative evaluations of the geo mechanical index. It is therefore very useful to know, for the specific problem, which parameters condition the stress and strain evolution in the rock around the tunnel and in the supports to a greater extent so as to be able to direct any further investigations towards the definition of the most influential parameters. 


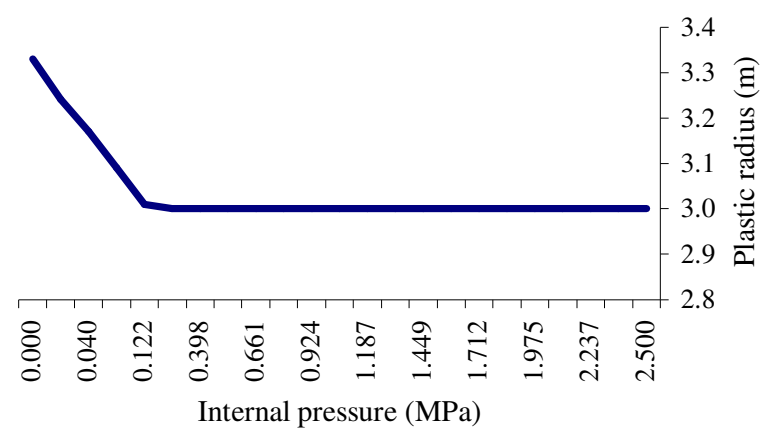

Fig. 13: Trend of the plastic radius $R_{p l}$ in function of the internal pressure $\mathrm{p}$ (case $\mathrm{b}$ )

(a)

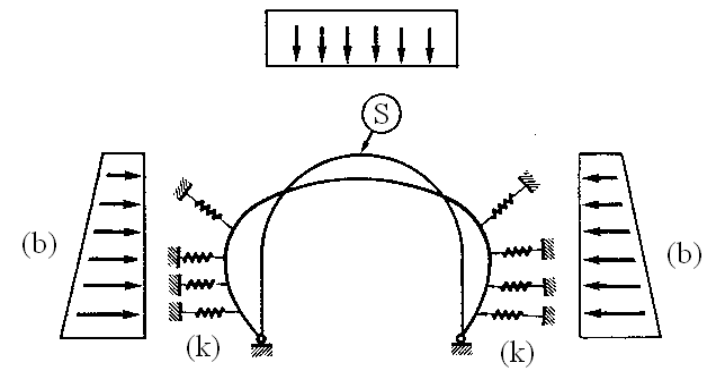

Fig. 14: Calculation scheme of the hyperstatic reaction method. Key: (a) vertical load, (b): Horizontal loads, (S): Support, (K): Support-rock connection spring

The convergence-confinement method is suitable for this purpose due to its working simplicity and calculation speed, being able to perform numerous analyses in short times changing one parameter at a time. More complex numerical methods instead require much longer calculation and interpretation times and can only be used for parametrical analysis limited to the most influential parameters.

Definition of the vertical load on the support structure: Some numerical calculation methods, for example the hyperstatic reaction method (Fig. 14), require the definition of the loads acting on the supports. The evaluation of the loads can be made using the convergence-confinement method ( $p_{\text {eq }}$ in Fig. 7) with the intersection between the convergenceconfinement curve of the tunnel and the reaction line of the support structures. The vertical load is usually assumed equal to $p_{e q}$, while the horizontal load is assumed equal to a certain fraction of the vertical load.

Initial estimation of the thickness of the plastic zones around the tunnel: Radial bolting is a widely used tunnel rock reinforcement technique. The evaluation of the length of each bolt is usually based on the thickness of the plastic zone around the void. It is usually necessary that the bolt should have an anchorage section (at least equal to (25-30\%) of its total length) that goes beyond the plastic zone, in rock with an elastic behaviour. The convergence-confinement method allows the immediate estimation of the thickness of the plastic zone and therefore also of the length of the radial bolting.

Evaluation of the entity of the stress and strain perturbation around the tunnel: The route of a tunnel often approaches already existing natural or man made underground works. In these cases it is necessary to know the reciprocal influence of the two works and the minimum distance that must be respected. It is easy to obtain the trend of the stresses and strains of a rock mass going away from the tunnel using the convergence-confinement method, taking into consideration the internal pressure produced by the support work. It is therefore possible to define the "limit distance" at which the stress-strain perturbation becomes negligible: A perturbation is therefore usually considered negligible when the difference in the absolute value between the radial or circumferential stress in the rock and the lithostatic stress $\mathrm{p}_{0}$ is lower than $(3-5 \%)$ of the $\mathrm{p}_{0}$.

Natural or man made voids that exist at a distance that is greater than the higher of the two limit distances (of the existing void or of the tunnel) do not constitute a serious problem for the tunnel.

The portion of rock that is "perturbed" by the construction of the tunnel is also the volume of rock that should be investigated using geognostic probes and geophysical tests, both before and during the construction of the tunnel.

Estimation of the entity of the tunnel convergences: It is necessary to estimate, already at the design stage, the entity of the convergences that a tunnel displays in order to:

- Decide on the type of measurement instrumentation to use and, in particular, on its precision

- Define beforehand the various degrees of attention and alarm, in relation to the convergence values that will be periodically measured

An approximate but quick estimation of the expected convergences $\Delta$ can be obtained using the convergenceconfinement method (Fig. 7). 
$\Delta=2 \cdot\left(\left|\mathrm{u}_{\mathrm{eq}}\right|-\left|\mathrm{u}_{\mathrm{in}}\right|\right)$

Optimisation of the support structures and the reinforcement elements of the rock: In the more complex cases, several rock support and rock reinforcement works are necessary to guarantee stability of the tunnel until the final lining is installed. The convergence-confinement method, because of its versatility and calculation simplicity, is well suited to look for the various support and rock reinforcement combinations that are necessary to ensure the stability of the tunnel with the same degree of safety. Many different analyses in which different types of support and different rock reinforcement intensities are foreseen are in fact necessary at this stage. The most advantageous support-reinforcement combination, from the economic point of view, between all the ones considered equivalent from the static point of view, is then chosen.

Back-analysis of the monitoring measurements: The monitoring measurements of a tunnel can play an important role on the calibration of the geomechanical parameters and therefore on the verification of the stability conditions of the support and rock reinforcement interventions during the work procedures. Back-analysis consists in obtaining the calibrated parameters of the rock which, inserted into the calculation model, allow one to obtain the same measured values. In order to perform a back-analysis it is usually necessary to define:

- A suitable calculation method that is able to determine the stress and strain state of the rock mass

- The error function, that represents the distance between the measurements that have been carried out on the site and the corresponding results obtained with the chosen calculation model:

$\varepsilon=\frac{1}{m} \cdot \sum_{j=1}^{m}\left[\frac{\bar{\eta}_{j}-f_{j}(x)}{\bar{\eta}_{j}}\right]^{2}=\frac{1}{m} \cdot \sum_{j=1}^{m}\left[1-\frac{f_{j}(x)}{\bar{\eta}_{j}}\right]^{2}$

Where:

$\varepsilon=$ The error function

$\bar{\eta}_{\mathrm{j}}=$ The generic measurement

$f_{j}=$ The function that is generally non linear and unknown in analytical terms, which connects the vector of the unknown parameters to the $\eta_{j}$ results of the calculation which corresponds to the $\mathrm{j}$ measurement $\mathrm{x}=$ Vector of the unknown parameters of the backanalysis procedure

$\mathrm{m}=$ Number of the available on site measurements

- An efficient logarithm that minimises the error function in relation to the unknown parameters

As numerous analyses are necessary during a backanalysis procedure, the calculation model of a tunnel should be quick and easy to interpret: The convergenceconfinement method is suitable for this purpose.

Evaluation of the static condition of the face: With the modern tendency of excavating full-face tunnels, even in poor quality grounds, the static conditions of the excavation face take on an increasingly important role. The stress and strain state of the face influences the choice of the types of reinforcement and support interventions that must be adopted to guarantee tunnel stability. Lunardi ${ }^{[14]}$ identified three possible conditions of an excavation face (Fig. 15):

- Stable excavation face: The rock has an elastic behaviour and the extrusion displacements are low (of a centimetre order as a maximum) the flow lines of the stresses are deviated through a natural arch effect that develops close to the face, if necessary, traditional support methods can be used (steel ribs, shotcrete, radial bolting)

- Stable excavation face in the short term: The rock presents elastic-plastic behaviour and the extrusions can reach a maximum order of magnitude of decimetres; the flow lines of the stresses are deviated at a certain distance from the face, beyond the plastic zone, traditional supports are necessary perhaps together with a slight reinforcement of the excavation face with VTR

- Unstable excavation face: The rock has an elasticplastic behaviour, with extensive plastic zones ahead of the face the extrusion displacements are very high, of the order of metres, a natural arch effect cannot be formed ahead of the face, apart from support works, reinforcement interventions of the nucleus and around the tunnel profile, ahead of the face, are also necessary

It has been shown, in Fig. 10 and 11, how it is easy to obtain an estimation of the extrusion of an excavation face and an evaluation of the existence and thickness of a plastic zone ahead of the face using the convergence-confinement method (Eq. 27-34). 


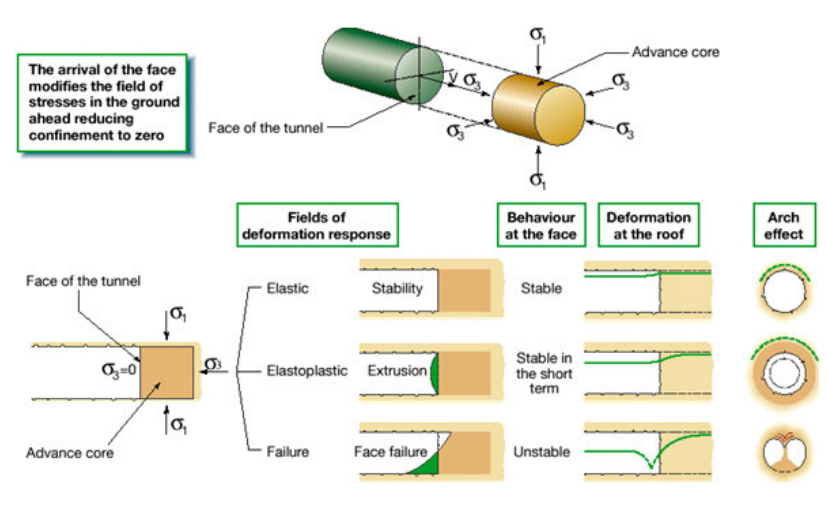

Fig. 15: Three possible types of behaviour of an excavation face without interventions: stable with elastic behaviour, stable in the short term with elastic-plastic behaviour and unstable ${ }^{[14]}$

In short, the convergence-confinement method is able to offer a preliminary evaluation of the static conditions of an excavation face and therefore an indication of the interventions that are necessary to make a tunnel stable.

\section{CONCLUSION}

The convergence-confinement method has been presented in this study with the two analytical solutions that have been most frequently used in recent years. The way of considering the support and rock reinforcement interventions using the method was then illustrated. The jointed analysis using the convergenceconfinement method of the circular geometry and of the spherical void was also presented. Finally, the ambits in the geo mechanical design of a tunnel, in which the convergence-confinement method still plays an important role today, in spite of the development and spread of more sophisticated numerical calculation methods, have been identified.

\section{REFERENCES}

1. Rechsteiner, G.F. and G. Lombardi, 1974. Une methode de calcul elasto-plastique de l'etat de tension et de deformation autour d'une cavite souterraine. Proceedings of the 3rd Congress on Advances in Rock Mechanics, International Society for Rock Mechanics, pp: 1049-1054.
2. Brown, E.T., J.W. Bray, B. Ladanyi and E. Hoek, 1983. Ground response curves for rock tunnels. J. Geotech. Eng., 109: 15-39. http://cedb.asce.org/cgi/WWWdisplay.cgi?8300093

3. Oreste, P.P., 2003. Analysis of structural interaction in tunnels using the convergenceconfinement approach. Tunnell. Underground Space Technol., 18: 347-363. http://www.ingentaconnect.com/content/els/08867 798/2003/00000018/00000004/art00004

4. Panet, M., 1995. Le calcul des tunnels par la méthode convergence-confinement. Presses de l'école nationale des Ponts et chaussées, Paris. ISBN: 2859782303, pp : 177.

5. Ribacchi, R. and R. Riccioni, 1977. Stato di sforzo e deformazione intorno ad una galleria circolare. Gallerie e Grandi Opere Sotterranee, 5: 7-18.

6. Hoek, E. and E.T. Brown, 1980. Underground Excavations in Rock. 1st Edn., The Institution of Mining and Metallurgy, London, ISBN: 0419 160302, pp: 527.

7. Panet, M. and A. Guenot, 1982. Analysis of convergence behind the face of a tunnel. Proceeding of the International Symposium Tunnelling, (IST'82), The Institution of Mining and Metallurgy, London, pp: 197-204.

8. AFTES., 2001. Recommandations on the convergence-confinement method.

http://www.aftes_asso.fr/publications_recommanda tions.html

9. Peila, D. and P.P. Oreste, 1995. Axisymmetrical analysis of ground reinforcing in tunnelling design. Comput. Geotech., 17: 253-274. http://www.ingentaconnect.com/content/els/02663 52x/1995/00000017/00000002/art93871

10. Oreste, P.P. and D. Peila, 1996. Radial passive rockbolting in tunnelling design with a new convergence-confinement model. Int. J. Rock Mec. Min. Sci., 33: 443-454.

http://www.ingentaconnect.com/content/els/01489 062/1996/00000033/00000005/art00009

11. Lembo-Fazio, A. and R. Ribacchi, 1986. Stato di sforzo e deformazione intorno ad una galleria. I Conferenza di Meccanica ed Ingegneria delle rocce, Politecnico di Torino, Italy, Nov. 25-28. 
12. Egger, P., 1974. Gebirgsdruck im tunnelbau und stutzwirkung der ortsbrust bei uberschreiten der gebirgsfestigkeit. Proceedings of the 3rd ISRM Congress, Denver, USA.

13. Bieniawski, Z.T., 1984. Rock mechanics design in mining and tunnelling. Bulkema, Rotterdam. http://infoscience.epfl.ch/record/27566
14. Lunardi, P., 2000. The design and construction of tunnels using the approach based on the analysis of controlled deformations in rocks and soils. http://www.rocksoil.com/p_d_f/t_and_t_rocksoil_s upp.pdf 